\title{
Risk of invasive pneumococcal infections among working age adults with asthma
}

\author{
Peter Klemets, ${ }^{1}$ Outi Lyytikäinen, ${ }^{1}$ Petri Ruutu, ${ }^{1}$ Jukka Ollgren, ${ }^{1}$ Tarja Kaijalainen, ${ }^{2}$ \\ Maija Leinonen, ${ }^{2} \mathrm{~J}$ Pekka Nuorti ${ }^{1}$
}

${ }^{1}$ National Institute for Health and Welfare (THL), Department of Infectious Disease Surveillance and Control, Helsinki, Finland

${ }^{2}$ National Institute for Health and Welfare, Lifecourse and Services Department, Finland

\section{Correspondence to}

J Pekka Nuorti, National Center for Immunization and

Respiratory Diseases, 1600 Clifton Road, NE, Mailstop C-23, Centers for Disease Control and Prevention (CDC), Atlanta, GA 30333, USA; pnuorti@cdc.gov

Received 6 December 2009 Accepted 12 May 2010

\section{ABSTRACT}

Background Information about the risk of invasive pneumococcal infection (IPI) among adults with asthma is limited and inconsistent. To evaluate this association, a population-based case-control study was conducted.

Methods Cases of IPI (Streptococcus pneumoniae isolated from blood or cerebrospinal fluid) were identified through national, population-based laboratory surveillance during 1995-2002. To maximise exclusion of chronic obstructive pulmonary disease, the analysis was limited to patients aged 18-49 years and 10 selected age-, sex- and health district-matched controls for each case from the Population Information System. Information on underlying medical conditions was obtained through linking surveillance data to other national health registries. Asthma requiring $\geq 1$ hospitalisation in the past 12 months was defined as high risk asthma (HRA); low risk asthma (LRA) was defined as entitlement to prescription drug benefits and no hospitalisation for asthma in the past 12 months.

Results 1282 patients with IPI and 12785 control subjects were identified. Overall, $7.1 \%$ of cases and $2.5 \%$ of controls had asthma $6.0 \%$ and $2.4 \%$ had LRA whereas $1.1 \%$ and $0.1 \%$ had HRA, respectively. After adjustment for other independent risk factors in a conditional logistic regression model, IPI was associated with both LRA (matched OR (mOR) 2.8; 95\% $\mathrm{Cl} 2.1$ to 3.6 ) and HRA (mOR, 12.3; 95\% Cl 5.4 to 28.0 ). The adjusted population-attributable risk was $0.039(95 \%$ $\mathrm{Cl} 0.023$ to 0.055 ) for LRA and 0.01 (95\% Cl 0.0035 to 0.017) for HRA.

Conclusions Working age adults with asthma are at increased risk of IPI. In this population, 5\% of disease burden could be attributed to asthma. These findings support adding medicated asthma in adults to the list of indications for pneumococcal vaccination.

Streptococcus pneumoniae is a leading cause of serious illness, particularly among those with certain underlying medical conditions. Chronic obstructive pulmonary diseases (COPDs) are established risk factors for invasive pneumococcal infection (IPI), ${ }^{1}$ but there has been uncertainty as to whether persons with asthma without prolonged oral corticosteroid use are at increased risk and if they should be vaccinated with pneumococcal polysaccharide vaccine (PPV23). ${ }^{1}$ Available information regarding asthma and risk of IPI is limited and somewhat inconsistent. ${ }^{2} 3$ To evaluate the association between asthma and IPI among adults aged 18-49 years, we conducted a nationwide, registryand population-based case-control study.

\section{METHODS}

\section{Surveillance}

In Finland (population, 5.2 million) all clinical microbiology laboratories, including private laboratories, notify (generally electronically) all bacterial isolations from blood and cerebrospinal fluid (CSF), including $S$ pneumoniae, to the National Infectious Disease Registry (NIDR) which is maintained by the National Institute of Health and Welfare (THL). Each of these mandatory notifications includes information on the causative microbe, date and type of specimen, date of birth, sex and the patient's place of treatment (healthcare facility).

\section{Ascertainment of cases and controls}

A case of IPI was defined as an illness in which $S$ pneumoniae was isolated from blood and/or CSF from 1 January 1995 to 31 December 2002. Among the overall 4611 episodes of IPI for whom National Identity Codes were available at the primary diagnostic laboratory, 4357 (94.5\%) first episodes of disease were identified. The 1282 (29.4\%) patients who were aged $18-49$ years were included in the case-control study. As the differential diagnosis of asthma and COPD in older adults is difficult, we restricted our study population to persons aged 18-49 years to maximise exclusion of COPD and to reduce confounding from cigarette smokingrelated lung disease. ${ }^{2}$ For each patient, 10 controls matched on age (year of birth \pm 1 year), sex and health district of residence were randomly selected from the National Population Information System.

Definitions and identification of underlying medical conditions

Information on underlying medical conditions for patients and controls was obtained by linking the case-control study database to four national, population-based healthcare registries by using each individual's unique National Identity Code. These databases included the National Social Insurance Institution (NSII), the National Hospital Discharge Register (HILMO), the Cancer Registry and the NIDR. The patient's date of the first positive culture for $S$ pneumoniae was used as the reference date for cases and their respective controls.

\section{Definitions of asthma and exclusion of COPD}

Patients and controls with asthma were defined as persons who had been granted a prescription drug benefit for asthma or COPD by the NSII, or who had a record of hospitalisation for these conditions during 12 months before the reference date in the HILMO database. High risk asthma (HRA) (International Classification of Diseases, 9th and 10th Revision (ICD-9, ICD-10) codes in appendix 1) was 
defined as a record in the HILMO of at least one hospitalisation with an ICD-coded primary diagnosis for asthma in the past 12 months. Persons with low risk asthma (LRA) were defined as those entitled to NSII prescription drug benefit and no hospitalisation for asthma during the 12 months before the reference date. Overall, 80 (84.2\%) of 95 patients and 308 (98.1\%) of 314 controls had a record of prescription drug benefit available in the NSII database; the rest were identified through hospital admission data. Only $4(0.3 \%)$ patients and none of controls had a record of hospitalisation for COPD in the past 12 months; these cases were excluded from the multivariable analysis.

The NSII uses standard criteria for determining the patient's eligibility for prescription drug benefit. For asthma and COPD, the criteria include a physician's certificate verifying the diagnosis and determining the severity of illness (using lung function tests, including sprirometry, and need for prescription medications for at least 6 months (appendix 2)). Although asthma is not distinguished from COPD in the NSII database because both conditions are assigned the same reimbursement group code, we considered our study population to consist of individuals with medicated asthma because of restriction to persons $<50$ years of age. ${ }^{2}$ Therefore, in the Results and Discussion sections the cases and controls with a prescription drug benefit are referred as having 'asthma'.

\section{Other medical conditions}

The presence of the following underlying medical conditions among patients and controls was defined as an NSII record indicating a prescription drug benefit for these conditions: diabetes mellitus, congenital or acquired immunodeficiency, rheumatic and other autoimmune diseases requiring immunosuppressive treatment, solid organ and bone marrow transplantation, cardiac failure and renal failure. Alcohol-related diseases, chronic liver diseases, diseases of the spleen and CSF leak were defined as records in the HILMO with one or more ICD-coded discharge diagnoses within 12 months before the reference date (appendix 1). Diagnosis of haematological malignant neoplasm within 5 years before the reference date and non-haematological malignancies within 1 year was obtained from the Cancer Registry, and infection with HIV from the NIDR.

\section{Serotyping of bacterial isolates}

$S$ pneumoniae isolates sent to the reference laboratory at the KTL as a part of national surveillance from 1995 to 2002 were serotyped as described previously. ${ }^{4}$ Pneumococcal serotypes were grouped as follows: 7-valent pneumococcal conjugate vaccine (PCV7) serotypes (4, 6B, 9V, 14, 18C, 19F and 23F); 10-valent (adding serotypes 1, 5 and $7 \mathrm{~F}$ ); 13-valent (adding serotypes 3, 6A and 19A); PPV23 serotypes (1, 2, 3, 4, 5, 6B, 7F, 8, 9N, 9V, 10A, 11A, 12F, 14, 15B, 17F, 18C, 19A, 19F, 20, 22F, 23F and 33F); and all other serotypes/groups.

\section{Statistical analysis}

For calculating the average annualised incidence of IPI among persons with asthma, the total number of persons $18-49$ years of age who had prescription drug benefits for asthma or COPD during 1995-2002 in the NSII database was used as the denominator. To identify independent factors associated with IPI and to control for confounding, we developed a conditional logistic regression model. Asthma was the main variable of interest; covariates included other underlying conditions for which PPV23 is recommended. ${ }^{1}$ Matched ORs (mORs) and 95\% CIs were calculated to compare risk factor characteristics between patients and controls. Adjusted population-attributable risks (PARs) for the independent risk factors in the model were calculated as described previously. ${ }^{5}$ PAR estimates the public health impact of a particular exposure - that is, the proportion of disease that could be prevented if the exposure was eliminated, expressed as a percentage. In the presence of confounding, the definition of PAR is: $\mathrm{Pd}(\mathrm{RR}-1) / \mathrm{RR}$, where $\mathrm{Pd}$ is the probability of exposure given disease and $R R$ is the multivariate relative risk, estimated here from the mOR. Data were analysed by using SPSS for Windows version 16.0 (SPSS, Chicago, Illinois, USA).

\section{Ethical aspects}

Research use of data collected in national registries was authorised by the Ministry of Social Affairs and Health, the Finnish Data Protection Authority and the National Research and Development Center for Welfare and Health.

\section{RESULTS}

\section{Characteristics of patients and controls}

During 1995-2002, national surveillance identified 1282 persons aged 18-49 years with IPI; these patients were included in the case-control study. The median age of patients and their controls was 38.0 years and $67.8 \%$ were male.

Overall, $359(28.0 \%)$ patients compared with $743(5.8 \%)$ of control subjects $(p<0.001)$ had an underlying medical condition for which the PPV23 is currently recommended by Finnish public health authorities for asthma; vaccine target groups are similar to those of the US Advisory Committee on Immunization Practices. ${ }^{1}$ Among the cases, asthma was the second most common underlying condition identified (7.1\%) following alcohol-related diseases (11.8\%), whereas among controls asthma was the most common underlying condition $(2.5 \%)$ followed by immunodeficiency or rheumatic diseases and diabetes mellitus (table 1). Overall, $9.4 \%$ of cases had a recognised condition or treatment leading to immunosuppression (immunodeficiency or rheumatic diseases, haematological and non-haematological malignancy, HIV infection or diseases of the spleen).

Of the cases, $77(6.0 \%)$ had LRA compared with 302 (2.4\%) of the controls; only $14(1.1 \%)$ patients and $12(0.1 \%)$ controls had HRA (table 1). The median duration of hospitalisation for the patients

Table 1 Underlying medical conditions among persons aged $18-49$ years with invasive pneumococcal disease and control subjects, Finland, 1995-2002

\begin{tabular}{lcr}
\hline Characteristic* & $\begin{array}{c}\text { Patients } \\
\text { (N=1282) }\end{array}$ & $\begin{array}{c}\text { Controls } \\
\text { (N=12785) }\end{array}$ \\
\hline Alcohol-related diseases & $151(11.8)$ & $72(0.6)$ \\
Asthma or COPD & $95(7.4)$ & $314(2.5)$ \\
$\quad$ HRA $\dagger$ & $14(1.1)$ & $12(0.1)$ \\
LRA & $77(6.0)$ & $302(2.4)$ \\
$\quad$ Hospitalisation for COPD & $4(0.3)$ & $0(0.0)$ \\
Diabetes mellitus & $45(3.5)$ & $148(1.2)$ \\
Haematological malignancy & $25(2.0)$ & $5(0.04)$ \\
Organ or bone marrow transplantation & $13(1.0)$ & $13(0.1)$ \\
Non-haematological malignancy & $11(0.9)$ & $23(0.2)$ \\
Immunodeficiency or rheumatic diseases & $56(4.4)$ & $190(1.5)$ \\
HIV infection & $10(0.8)$ & $7(0.1)$ \\
Chronic liver disease & $9(0.7)$ & $1(0.008)$ \\
Cardiac failure & $8(0.6)$ & $13(0.1)$ \\
Chronic renal failure & $6(0.5)$ & $4(0.03)$ \\
Diseases of spleen & $2(0.2)$ & $1(0.008)$ \\
No underlying condition & $923(72.0)$ & $12042(94.2)$ \\
\hline
\end{tabular}

*Individuals may have more than one underlying condition.

†Hospitalisation for asthma in the past 12 months.

‡Entitlement to prescription drug benefit for asthma or COPD but no hospitalisation for asthma in the past 12 months.

COPD, chronic obstructive pulmonary disease; HRA, high risk asthma; LRA, low risk asthma. 
with IPI with HRA was 8 days (range, 4-69 days). Of the 77 patients with LRA, 10 (13.0\%) had a current indication for PPV23: alcohol-related diseases $(n=5)$, immunodeficiency or rheumatic disease $(n=4)$, non-haematological malignancy $(n=2)$ and chronic liver disease $(n=1)$. No patients with HRA had other underlying conditions. During the 7 and 28 days after the first positive blood or CSF culture, $6 / 91(6.6 \%)$ and $7 / 91(7.7 \%)$ of patients with asthma versus $82 / 1187(6.9 \%)$ and $117 / 1187$ (9.9\%) of the other IPI cases died, respectively. All deaths were in the LRA group. The annual incidence of IPI among persons aged 18-49 years with LRA was estimated to be 18.2 cases/100 000 population.

S pneumoniae was isolated from blood for 1196 (93.3\%) patients and from CSF for $86(6.7 \%)$. Of the $1056(82.4 \%)$ isolates that were serotyped, serotypes 4 (20.8\%), 7F (12.1\%), 14 (9.7\%), 3 $(9.0 \%)$ and $9 \mathrm{~V}(7.7 \%)$ were the most common. Of the serotypes causing IPI, 90\% were covered in PPV23; 76.1\% were covered in the paediatric 13-valent pneumococcal conjugate vaccine (table 3). Serotypes in different pneumococcal conjugate vaccine formulations accounted for a larger number of isolates among patients with asthma compared with those without asthma.

\section{Multivariable analysis}

In a conditional logistic regression model adjusted for other risk factors, patients were more likely than controls to have HRA (mOR, 12.3; 95\% CI 5.4 to 28.0) and LRA (mOR, 2.8; 95\% CI 2.1 to 3.6) (table 2). The adjusted PARs for HRA and LRA were 1\% (0.01; $95 \%$ CI 0.0035 to 0.017$)$ and 4\% (0.039; 95\% CI 0.023 to $0.055)$, respectively. Inclusion of the four patients hospitalised for COPD and their controls in the model did not change the mORs for HRA or LRA (data not shown). We also constructed a second conditional logistic regression model which excluded patients who had immunosuppressive conditions and their controls. The results from this model were similar to the main model: the mOR for HRA was 15.6 (95\% CI 6.5 to 37.6) and that for LRA was 2.9 (95\% CI 2.2 to 3.9$)$.

\section{DISCUSSION}

Results of our national, population-based study indicate that LRA is also an independent factor moderately increasing the risk for IPI among adults 18-49 years of age. Persons with a recent hospitalisation for asthma (HRA) had over a fourfold increased risk of IPI compared with those who had not required hospital care (LRA). Overall, an estimated $5 \%$ of the IPI disease burden in the working age adult population was attributable to asthma.
The prevalence of asthma continues to increase globally, ${ }^{67}$ and in the USA 16 million (7.3\%) adults are estimated to have asthma. ${ }^{8}$ Even though accurate assessment of the prevalence is complicated by varying definitions of asthma, an estimated $4.4 \%$ of Finnish adults have asthma and the prevalence has increased since the 1960s. ${ }^{9}{ }^{10}$ In our study, asthma was the second most common underlying condition among patients with IPI (7.1\% of cases). Our findings therefore have important public health implications and support adding medically treated asthma in adults to the list of indications for pneumococcal vaccination which already includes other chronic lung diseases such as COPD and emphysema.

Results from previous studies on whether or not asthma is associated with IPI are limited and have been inconsistent. ${ }^{1-3}$ No previous data are available from European countries. A recent study among persons aged 2-49 years enrolled in the Medicaid programme in one US state, where cases of IPI identified through active population- and laboratory-based surveillance were linked to administrative data to determine the presence of asthma, suggested that asthma moderately increased the risk of IPI. ${ }^{2}$ However, another recent study in mostly older adults (average age $>50$ years) analysed administrative data from the US Veteran's Administration Health Care system and found that compared with control subjects, persons with COPD had an increased risk of hospitalisation for pneumococcal pneumonia but persons with asthma did not. ${ }^{3}$ The differences in these study results may be related to the differences in data sources, study populations and their age, definitions used for asthma, outcomes evaluated (IPI being more specific than ICD-coded pneumococcal pneumonia) and analytical methods.

Overall, the findings of our study are largely consistent with the US Medicaid database study, ${ }^{2}$ in which persons who had asthma had an overall adjusted OR of 2.4 for IPI compared with control subjects without asthma. However, there were also some important differences. In the US study, high risk patients were defined as those requiring at least one of the following: admission to hospital or emergency department, the use of intensive treatment for acute asthma attacks, long-term corticosteroid use or the dispensing of $\geq 3$ prescriptions for $\beta$-agonists within the year before enrolment in the study. ${ }^{2}$ These high risk patients accounted for $83 \%$ of IPI cases with asthma in the study. Although the OR for HRA (2.6) in the US Medicaid study was not different from the overall OR (2.4), the annual incidence of IPI was estimated to be almost twofold (4.2 vs 2.3 per 10000 persons) among patients with HRA compared with those with LRA. The association of IPI with LRA

Table 2 Association of asthma with invasive pneumococcal infection among persons aged 18-49 years: a conditional logistic regression model

\begin{tabular}{|c|c|c|c|c|c|c|c|}
\hline Characteristic & $\begin{array}{l}\text { Patients } \\
\text { ( } N=1282) \\
n(\%)\end{array}$ & $\begin{array}{l}\text { Controls } \\
(\mathrm{N}=12785) \\
n(\%)\end{array}$ & $\mathrm{mOR}$ & $95 \% \mathrm{Cl}$ & p Value & PAR $^{*}$ & $95 \% \mathrm{Cl}$ \\
\hline High risk asthma† & $14(1.1)$ & $12(0.1)$ & 12.3 & 5.4 to 28.0 & $<0.001$ & 0.01 & 0.0035 to 0.017 \\
\hline Chronic liver disease & $9(0.7)$ & $1(<0.1)$ & 96.6 & 10.6 to 883.9 & $<0.001$ & 0.0069 & 0.0016 to 0.012 \\
\hline Haematological malignancy & $25(2.0)$ & $5(<0.1)$ & 56.0 & 20.0 to 157.0 & $<0.001$ & 0.019 & 0.010 to 0.028 \\
\hline Diseases of spleen & $2(0.2)$ & $1(<0.1)$ & 35.6 & 3.0 to 428.5 & $<0.005$ & 0.0015 & 0.00 to 0.0040 \\
\hline Non-haematological malignancy & $11(0.9)$ & $23(0.2)$ & 5.1 & 2.4 to 10.9 & $<0.001$ & 0.0069 & 0.0016 to 0.013 \\
\hline Organ or bone marrow transplantation & $13(1.0)$ & $13(0.1)$ & 2.9 & 1.0 to 8.8 & 0.059 & 0.0067 & 0.00 to 0.015 \\
\hline Cardiac failure & $8(0.6)$ & $13(0.1)$ & 2.8 & 1.0 to 8.2 & 0.059 & 0.004 & 0.00 to 0.010 \\
\hline Immunodeficiency or rheumatic diseases & $56(4.4)$ & $190(1.5)$ & 3.1 & 2.3 to 4.4 & $<0.001$ & 0.030 & 0.017 to 0.044 \\
\hline Diabetes mellitus & $45(3.5)$ & $148(1.2)$ & 2.3 & 1.6 to 3.5 & $<0.001$ & 0.020 & 0.0076 to 0.034 \\
\hline
\end{tabular}

*Population-attributable risk. The population exposure prevalence is estimated internally from cases-that is, 1.1\% for HRA and 6.0\% for LRA.

†Hospitalisation for asthma in the past 12 months; four patients hospitalised for chronic obstructive pulmonary disease and their controls were excluded.

$\neq$ Entitlement to a prescription drug benefit for asthma but no hospitalisation for asthma in the past 12 months.

$\mathrm{mOR}$, matched odds ratio. 
Table 3 Pneumococcal serotypes causing invasive infections among adults $18-49$ years of age with and without asthma, Finland, 1995-2002

\begin{tabular}{llll}
\hline & $\mathbf{n}$ (\%) & & \\
\cline { 2 - 4 } & $\begin{array}{l}\text { Asthma } \\
\mathbf{N = 8 1}(\mathbf{7 . 7 )}\end{array}$ & $\begin{array}{l}\text { No asthma } \\
\mathbf{N = 9 7 5}(\mathbf{9 2 . 3})\end{array}$ & $\begin{array}{l}\text { Total } \\
\mathbf{N = 1 0 5 6}\end{array}$ \\
\hline Serotypes covered in vaccine formulations & & \\
7-valent PCV & $44(54.3)$ & $468(48.0)$ & $512(48.5)$ \\
10-valent PCV & $59(72.8)$ & $610(62.6)$ & $669(63.3)$ \\
13-valent PCV & $64(79.0)$ & $740(75.9)$ & $804(76.1)$ \\
23-valent PPV & $78(96.3)$ & $903(92.6)$ & $981(92.9)$ \\
23-valent PPV† & $73(90.1)$ & $855(87.7)$ & $928(87.9)$ \\
\hline
\end{tabular}

*All serogroups included in the PPV23 vaccine.

†Not including serogroups 10,11, 12, 15, 17 and 33 which were not serotyped. During 1995-1999, serogroups $10,11,12,15,17$ and 33 were not identified to type level; during 2000-2002, serogroups 11,12 and 15 were identified to type level.

alone was not significant, suggesting that patients with HRA may have been the major contributor to the overall association. ${ }^{2}$ In our study, diagnosis of asthma in the low risk group had been established by lung function tests, and all persons with HRA had had at least one hospitalisation for asthma during the 12 months before the IPI episode. Although the OR among persons with HRA was fourfold higher than among persons with LRA, persons with HRA accounted for only $\sim 15 \%$ of all cases with asthma and, consequently, the PAR was small (1\%). Our findings emphasise the importance of taking into account the variability in risk of IPI among persons with asthma of different severity resulting from the type and intensity of treatments (immunosuppression) and the need for hospitalisation.

Because of lack of a standard definition for paediatric asthma, diagnostic difficulties and the fact that many children outgrow wheezing, we excluded children from our study. The inclusion of children aged 2-17 years in the US Medicaid study ${ }^{2}$ may have further contributed to some of the observed differences, although for the age group 18-49 years the ORs were similar to those of our study. Finally, our study base was nationwide, population based and unselected, whereas the US study included mostly persons of low socioeconomic status enrolled in a Medicaid (TennCare) programme and may not be fully generalisable to the general US population or to other countries.

In asthma, several pathological alterations in the airways could be associated with increased risk of IPI. The mucociliary clearance of the bronchi is impaired due to abnormal production of sputum and airway obstruction. ${ }^{11}$ These abnormalities can serve as a focus for viral infections which might predispose to development of invasive bacterial infections. ${ }^{12-15}$ Long-term immunosuppressive treatment of asthma with corticosteroids may be associated with increased risk for pneumococcal disease. ${ }^{1}$

Several limitations need to be considered in interpreting our findings. First, due to the registry-based study design, it was not possible to obtain information about the cigarette smoking status of the study subjects, which may be an important confounding factor. ${ }^{16}$ The US Medicaid study ${ }^{2}$ had only limited information about cigarette smoking, retrieved from inpatient discharge files, resulting in an underestimation of the prevalence of cigarette smoking in the study population and possibly to some residual confounding. Another possible confounder in studies assessing factors associated with IPI might be undiagnosed HIV infection. ${ }^{17} 18$ In Finland, however, the prevalence of HIV infection and the proportion of IPI cases who have HIV is very low and unlikely to have an effect on the study findings. ${ }^{19}$ In addition, we did not have information on socioeconomic status, which could be another possible confounder.
Secondly, although among the national registries we used to obtain data on co-morbidities for IPI cases, the Finnish Cancer Registry has almost $100 \%$ coverage ${ }^{20} 21$ and the comprehensiveness of the National Hospital Discharge Register data has been validated previously, ${ }^{22} 23$ it is well known that ICD coding in hospital discharge data may be incomplete and could be subject to misclassification. For this reason, we used hospital discharge data only to identify those underlying conditions for which data were not available in the two other registries (National Social Insurance Institution and Cancer Registry) which use standardised criteria and definitions. Differential diagnosis between reactive airway disease and COPD among middle-aged adults may be difficult and these syndromes may overlap. ${ }^{24}$ Because of the shared reimbursement code, we were unable to distinguish unequivocally between asthma and possible COPD among those who were classified as having LRA, and some misclassification among the two disease entities is possible. ${ }^{25}$ However, the number of IPI cases who may have had COPD in the absence of asthma is likely to be very small in our working age study population with a median age of 38 years. $^{29}$ In addition, misclassification of possible COPD as asthma would probably be non-differential and would therefore make detecting an association more difficult.

Thirdly, we had no information regarding the PPV23 vaccination status of study subjects. However, a recent nationally representative survey among persons 30 years of age and older in Finland found that uptake of PPV23 was extremely low (3\%) in the high risk target groups ${ }^{26}$ and only $2.6 \%$ of persons aged 18-64 years with asthma or COPD had ever received PPV23 (National Institute for Health and Welfare, unpublished data) making it unlikely that PPV23 vaccination influenced our findings. Fourthly, information on the specific medications of the patients and controls was not available. During 1997-2002, $91.2 \%$ of patients $18-49$ years of age who were entitled to prescription drug benefits (ie, the LRA group) received a prescription for inhalation and/or oral steroids; $16.9 \%$ received oral steroids (NSII, unpublished data).

This population-based study provides further evidence to support an association between asthma and IPI and suggests that, in addition to persons who require hospitalisation and prolonged oral corticosteroid treatment for HRA, those with LRA are also at increased risk of IPI, supporting adding medically treated asthma in adults to the list of indications for pneumococcal vaccination.

Acknowledgements Mikko Virtanen, MSc, is acknowledged for statistical advice.

Competing interests PK received a fee on two occasions (2006 and 2008) for giving a lecture at a symposium organised by Wyeth Finland. The other authors have no competing interests.

Provenance and peer review Not commissioned; externally peer reviewed.

\section{REFERENCES}

1. CDC. Prevention of pneumococcal disease: recommendations of the Advisory Committee on Immunization Practices (ACIP). MMWR Recomm Rep 1997:46:1-24

2. Talbot TR, Hartert TV, Mitchel E, et al. Asthma as a risk factor for invasive pneumococcal disease. N Engl J Med 2005;352:2082-90.

3. Lee TA, Weaver FM, Weiss KB. Impact of pneumococcal vaccination on pneumonia rates in patients with COPD and asthma. J Gen Intern Med 2007;22:62-7.

4. Kilpi T, Herva E, Kaijalainen T, et al. Bacteriology of acute otitis media in a cohort of Finnish children followed for the first two years of life. Pediatr Infect Dis J 2001;20:654-62

5. Bruzzi P, Green SB, Byar DP, et al. Estimating the population attributable risk for multiple risk factors using case-control data. Am J Epidemiol 1985;122:904-14.

6. Partridge MR. Asthma: 1987-2007. What have we achieved and what are the persisting challenges? Prim Care Respir J 2007;16:145-8.

7. Mannino DM, Homa DM, Akinbami LJ, et al. Surveillance for asthma-United States, 1980-1999. MMWR Surveill Summ 2002;51:1-13.

8. CDC. Influenza vaccination coverage among persons with asthma-United States, 2005-06 influenza season. MMWR Morb Mortal Wkly Rep 2008;57:653-7. 
9. Hedman J, Kaprio J, Poussa T, et al. Prevalence of asthma, aspirin intolerance, nasal polyposis and chronic obstructive pulmonary disease in a population-based study. Int J Epidemiol 1999;28:717-22.

10. Latvala J, von Hertzen $\mathrm{L}$, Lindholm $\mathrm{H}$, et al. Trends in prevalence of asthma and allergy in Finnish young men: nationwide study, 1966-2003. BMJ 2005;330:1186-7.

11. Fahy JV, Corry DB, Boushey HA. Airway inflammation and remodeling in asthma. Curr Opin Pulm Med 2000;6:15-20.

12. Bardin PG, Fraenkel DJ, Sanderson G, et al. Amplified rhinovirus colds in atopic subjects. Clin Exp Allergy 1994:24:457-64.

13. Koehler DR, Downey GP, Sweezey NB, et al. Lung inflammation as a therapeutic target in cystic fibrosis. Am J Respir Cell Mol Biol 2004;31:377-81.

14. Patel IS, Seemungal TA, Wilks M, et al. Relationship between bacterial colonisation and the frequency, character, and severity of COPD exacerbations. Thorax 2002;57:759-64.

15. Message SD, Johnston SL. Host defense function of the airway epithelium in health and disease: clinical background. J Leukoc Biol 2004;75:5-17.

16. Nuorti JP, Butler JC, Farley MM, et al. Cigarette smoking and invasive pneumococcal disease. Active Bacterial Core Surveillance Team. N Engl J Med 2000:342:681-9.

17. Cotton D. Asthma and invasive pneumococcal disease. N Engl J Med 2005:353:738-9; author reply 738-9.

18. Nuorti JP, Butler JC, Breiman RF. Smoking and pneumococcal disease. N Engl J Med 2000;343:219-20.

19. Klemets $\mathbf{P}$, Lyytikainen 0 , Ruutu $\mathrm{P}$, et al. Invasive pneumococcal infections among persons with and without underlying medical conditions: implications for prevention strategies. BMC Infect Dis 2008;8:96.

20. Teppo L, Pukkala E, Lehtonen M. Data quality and quality control of a populationbased cancer registry. Experience in Finland. Acta Oncol 1994;33:365-9.

21. Brenner $\mathbf{H}$, Hakulinen T. Reduction in selective under-ascertainment bias in population-based estimates of cancer patient survival by age adjustment. Eur $\mathrm{J}$ Cancer 2005; $41: 1788-93$.

22. Mahonen M, Salomaa V, Brommels M, et al. The validity of hospital discharge register data on coronary heart disease in Finland. Eur J Epidemiol 1997;13:403-15.

23. Pajunen $\mathbf{P}$, Koukkunen $\mathrm{H}$, Ketonen $\mathbf{M}$, et al. The validity of the Finnish Hospital Discharge Register and Causes of Death Register data on coronary heart disease. Eur $J$ Cardiovasc Prev Rehabil 2005;12:132-7.

24. Buist AS, McBurnie MA, Vollmer WM, et al. International variation in the prevalence of COPD (the BOLD Study): a population-based prevalence study. Lancet 2007:370:741-50.

25. Halbert RJ, Natoli JL, Gano A, et al. Global burden of COPD: systematic review and meta-analysis. Eur Respir J 2006;28:523-32.

26. Ruutu P, Kuusi M, Nuorti PJ, et al. Communicable diseases. In: Aromaa A, Koskinen S, eds. Health and functional capacity in Finland. Baseline results of the Health 2000 examination survey. Helsinki: National Public Health Institute, 2004:67-9.

\section{APPENDIX 1: ICD-9* AND ICD-10† CODES USED IN DEFINING UNDERLYING CONDITIONS FOR DATA IN THE NATIONAL HOSPITAL DISCHARGE DATABASE}

\begin{tabular}{|c|c|c|}
\hline Underlying condition & ICD-9 codes & ICD-10 codes \\
\hline $\begin{array}{l}\text { Alcohol-related diseases } \\
\text { (Morbus Wernicke, } \\
\text { mental, behavioural and } \\
\text { neurological disorders } \\
\text { due to alcohol use, } \\
\text { hepatic and pancreatic } \\
\text { disorders due to alcohol } \\
\text { use, toxic effects of } \\
\text { alcohol, maternal and } \\
\text { fetal care due to alcohol } \\
\text { abuse, rehabilitation and } \\
\text { counselling due to alcohol } \\
\text { abuse) }\end{array}$ & $\begin{array}{l}\text { 2651X; 2910A, 2911A, } \\
\text { 2913A, 2918A, 2948X, } \\
\text { 3039X, 3050A; 3318X; } \\
\text { 3451A; 3575A; 3594A; } \\
\text { 5710A, 5711A, 5712A, } \\
\text { 5713X; 5770D, 5771B, } \\
\text { 5771C, 5771D; 9800A, } \\
\text { 9801A, 9802A, 9803A, } \\
\text { 9808X, 9809X; 6554A; } \\
\text { 7607A, E850A; V654A }\end{array}$ & $\begin{array}{l}\text { E51.2; F10; G31.2; G40.51; } \\
\text { G62.1; G72.1; K70; K86; T51; } \\
\text { 035.4; P04.3; X45; Y91; } \\
\text { Z50.2; Z71.4 }\end{array}$ \\
\hline Asthma bronchiale & $\begin{array}{l}\text { 4930A, 4930B, 4931A, } \\
4931 B, 4939 A, 4939 B\end{array}$ & $\mathrm{~J} 45, \mathrm{~J} 46$ \\
\hline $\begin{array}{l}\text { Cerebrospinal fluid } \\
\text { leakage }\end{array}$ & $3498 \mathrm{~A}$ & G96.0 \\
\hline $\begin{array}{l}\text { Chronic liver } \\
\text { diseases (hepatic failure, } \\
\text { cirrhosis of liver and } \\
\text { biliary ducts, portal } \\
\text { hypertension, hepatorenal } \\
\text { syndrome and unspecified } \\
\text { liver disease) }\end{array}$ & $\begin{array}{l}\text { 5709A, 5719X, 5739X; } \\
\text { 5715A, 5716A, 5716X; } \\
\text { 5719X; 5724A; 5739X }\end{array}$ & K72; K74; K76.7; K76.9 \\
\hline COPD & $4912 B, 4960 A$ & J44 \\
\hline Diseases of the spleen & $2894 \mathrm{~A}, 2895 \mathrm{~A}$ & D73 \\
\hline
\end{tabular}

*International Classification of Diseases, 9th Revision.

t10th Revision.

COPD, chronic obstructive pulmonary disease.
APPENDIX 2: NATIONAL CRITERIA FOR PRESCRIPTION DRUG BENEFITS FOR ASTHMA OR COPD (REIMBURSEMENT OF MEDICATION COST), NATIONAL SOCIAL INSURANCE INSTITUTION (KELA), FINLAND ONE OF THE FOLLOWING CRITERIA SHOULD BE MET:

Asthma:

1. Diurnal variation in peak expiratory flow (PEF) of $>20 \%$ over 2 weeks

2. Increase of at least $12 \%$ and $200 \mathrm{ml}$ in PEF or forced expiratory volume in $1 \mathrm{~s}$

$\left(\mathrm{FEV}_{1}\right)$ in spirometry after bronchodilator use

3. Increase of at least $15 \%$ and $60 \mathrm{l} / \mathrm{min}$ (adults) in PEF after bronchodilator use

4. In the corticosteroid test, increase of at least $15 \%$ and $200 \mathrm{ml}$ in $\mathrm{FEV}_{1}$ or increase of at least $20 \%$ of average PEF measurements during several days

5. Severe or intermediate bronchial reactivity after histamine or metacholine challenge

6. Decrease of at least $15 \%$ of $\mathrm{PEF}$ or $\mathrm{FEV}_{1}$ during exercise test.

COPD:

1. $\mathrm{FEV}_{1}$ is permanently $<40 \%$ of the reference value or

2. $\mathrm{FEV}_{1}$ is permanently $<50 \%$ of the reference value and the need of hospitalisation due to exacerbation of COPD during bronchodilator use or at least two episodes of exacerbation requiring oral corticosteroid or antibiotics.

Drugs included in the prescription drug benefit (reimbursement of cost) for patients with asthma or COPD (2002)

1. Corticosteroids for systemic use

2. Adrenergics, inhalants

3. Inhaled glucocorticoids

4. Inhaled anticholinergics

5. Antiallergica, inhalants (cromoglicic acid and nedocromil)

6. Antiasthmatics for systemic use (excluding glucocorticoids) 